\title{
An Algorithm for Seamless Image Stitching and Its Application
}

\author{
Jing Xing, Zhenjiang Miao, and Jing Chen \\ Institute of Information Science, Beijing JiaoTong University, \\ Beijing 100044, P.R. China
}

\begin{abstract}
Panoramic image stitching used to create virtual environments for many applications is crucial for IBR. In this paper, we introduce a four-step algorithm based on SIFT features which makes some improvements on large exposure difference, large scene motion or other misregistrations when stitching panoramic images. We introduce a new method that is to add the adjacent images according to a weight function to eliminate the seam in the transition region. As a result, we apply the panoramic image to a computer game to enhance the reality of the game environment. Through lots of analyses and experiments we find that our method is simple and effective. Because this method is not sensitive to ordering, orientation, scaling and illumination, its stitching precision is much higher than many other methods.
\end{abstract}

Keywords: Homography, game environment, SIFT features, panorama.

\section{Introduction}

Because of the limitation of geometry-based Rendering, Image-based Rendering (IBR) is becoming more and more important. Panoramic image stitching is to merge a sequence of images with limited overlapping area into one blended picture. As a result, the panoramic images are applied to construct virtual environment which can get higher reality. Satisfying image stitching results are crucial, which means a natural transition from one image to another, both in structure and intensity within and possibly beyond the overlapping region.

There are many algorithms to stitching panoramic images. Feature-based methods begin by establishing correspondences between points, lines or other geometrical entities. The approach based on area [2] calculates the difference of pixel values in the searching region between two adjacent images. This method is easy to implement but the results are related to the illumination and orientation of the input images. There is another approach based on feature seam [2] that is to search a seam where adjacent images can be stitched. In this method, better results are gotten, but the results are not reliable. In this paper, the algorithm based on SIFT features and a new approach to eliminate the seam between adjacent images are introduced. 


\section{Algorithm Details of Images Stitching}

It's a key to match features in the images stitching. Among the local descriptors compared, SIFT features generally perform the best [1] [4]. Our algorithm is divided four steps and the details are shown as follow.

\subsection{Feature Matching}

Because larger motions, or matching collections of images where the geometric relationship between them is unknown, it is a key and difficulty to attract features of the images. In our experiment, we use SIFT features [4] and get good results.

SIFT features provide a set of features of an object that are not affected by many of the complications experienced in other methods, such as object scaling and rotation. To aid the extraction of these features the SIFT algorithm applies a four-stage filtering approach [5]: (1). Scale-Space Extremum Detection; (2). Key point Localization; (3). Orientation Assignment; (4). Key point Descriptor.

\subsection{Homography Calculating}

As the above described, given two images taken from the same viewpoint but in different directions, the relationship of the two images can be described by a planar projective motion model which is Homography metrics denoted $\mathrm{H}$. $\mathrm{H}$ is a matrix of $3 \times 3$, and we need four pairs matching points at least to calculate $H$. This projective transformation warps an image into another one using $x_{i}{ }^{\prime} \sim \mathrm{H} x_{i}$. To calculate $\mathrm{H}$ correctly is crucial in the images matching. In our experiment, we use the method of direct linearity transformation (DLT) [3] and at least four pairs' interest points from image 2 to image 1 are needed.

\subsection{Image Stitching}

The third step is to stitch the images. After calculating $\mathrm{H}$, image 2 is transformed into the same coordinate system as image 1 according the formulas introduces in section 2.2 [5]. Lots of information of image can be lost due to the transformation. In this experiment, we record the coordinates of the image 2 after transformation, and then stitch the two images directly. Described in formula is shown as follow:

$$
\left[\begin{array}{llll}
x 1 & x 2 & x 3 & x 4 \\
y 1 & y 2 & y 3 & y 4
\end{array}\right] \times H=\left[\begin{array}{cccc}
x 1^{\prime} & x 2^{\prime} & x 3^{\prime} & x 4^{\prime} \\
y 1^{\prime} & y 2^{\prime} & y 3^{\prime} & y 4^{\prime}
\end{array}\right]
$$

Where $\left[\begin{array}{llll}x 1 & x 2 & x 3 & x 4 \\ y 1 & y 2 & y 3 & y 4\end{array}\right]$ is a matrix of four corners' coordinates of image 2 (shown clockwise), and then we can get the four corners' coordinates of image 2 after transformation $\left[\begin{array}{llll}x 1^{\prime} & x 2^{\prime} & x 3^{\prime} & x 4^{\prime} \\ y 1^{\prime} & y 2^{\prime} & y 3^{\prime} & y 4^{\prime}\end{array}\right]$. 
According to the coordinates, we can stitch adjacent images exactly. Using this method, complex algorithm and unnecessary calculation are avoided. The illumination of the two original images is different, but the stitching result is not affected because of the SIFT features' insensitivities to illumination, oriental and scale.

\subsection{Eliminating the Seam of the Image After Stitching}

Although images can be stitched together exactly, a seam is still obvious in the transition region. There are many approaches to image stitching in the literature. Optimal seam algorithms [6] search for a curve in the overlap region on which the differences between image 1 and image 2 are minimal. Then, each image is copied to the corresponding side of the seam. However, the seam is visible when there is no such curve. Another approach is to minimize seam artifacts by smoothing the transition between the images. A related approach was suggested in [7], where a smooth function was added to the input images to force a consistency between the images in the seam curve. In case there are misalignments between the images [8], these methods leave artifacts in the mosaic such as double edges.

Our method is different from these methods: first, the camera parameters are not needed; second, our method can detect the seam between the two images correctly. Camera calibration is a complex work in big environment and the features are not easy to get. Our method avoids this problem. Because the algorithm based on SIFT features is adopted in our experiment, image 1 and image 2 are in the same coordinates system after image 2 multiplying $\mathrm{H}$, where $\mathrm{H}$ is the homography of the two images. In the same way, the transition region can be located easily.

In order to make the image look no visible seam, process the transition region naturally stitch is important. Our method mainly processes the transition region.

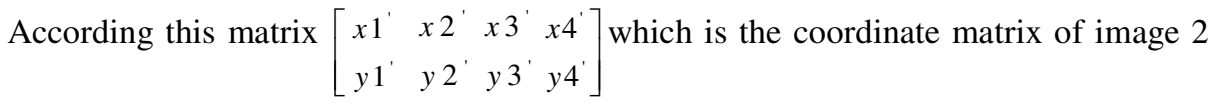
after transformation, the transition region is computed correctly, shown in figure 1:
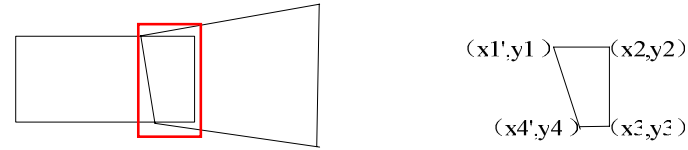

Fig. 1. The image after pasting two images (transition region in the red rectangle)

Because the transition region is the mix part of Image 1 and Image 2, we can assume a weight function and make the image after stitching smoothing. The image after stitching is noted as I. The left part of transition region is the image 1 and the right part is Image 2. Let the two images multiple a weight function $w(x)$ to eliminate the seam 
in transition region, and the transition region and weight function can be denoted as following formulas:

$$
\left\{\begin{array}{l}
S=(1-w(x)) * I 1(x, y)+w(x) * I 2(x, y) \\
w(x)=\sin (x * \pi /(2 * d))
\end{array}\right.
$$

where $0 \leq w(x) \leq 1 \quad 0 \leq x \leq d \quad d$ is the width of transition region and it can be computed according to the figure 1 .

\section{Experiment Results and Application}

In this experiment, the SIFT features are extracted from a pair of adjacent images. The features are matched using k-d tree, and the result is shown in figure 2 . In figure 2, two original images are given which have overlapping region. And then, we detect the SIFT features of the two images and match them. After detecting the features and calculating the homography of two adjacent images, we stitch the images by recording coordinates of the image after transformation. Another result is also given that is obtained by the method based on area. Form these results compared, higher accuracy is gotten in our method. There is a visible seam in the transition area after stitching. The seam in transition region is eliminated by using our method and the result is shown in figure 3 .
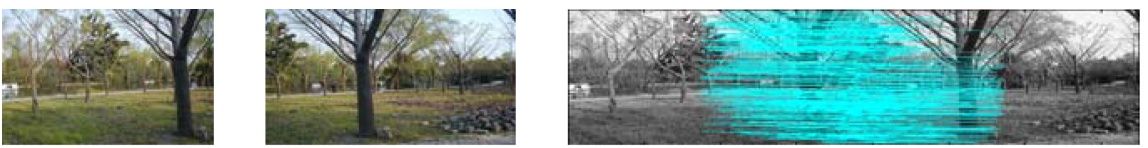

Fig. 2. Original images (the left two images) and SIFT feature matches extracted from a pair of images (right)
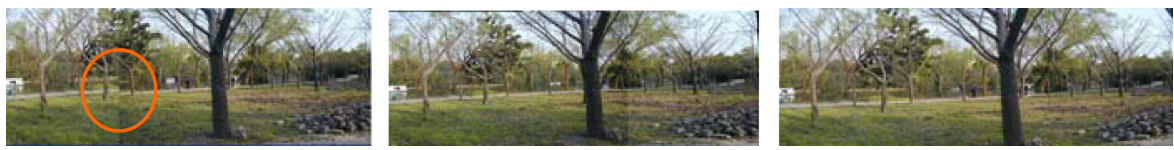

Fig. 3. the image after stitching using the method based on area [2] (the matching error is marked with red rectangle) and using the method based on SIFT features with obvious seam (middle) stitching result after smoothing the transition (right)

Some experiment results based on SIFT features are also shown as follow.
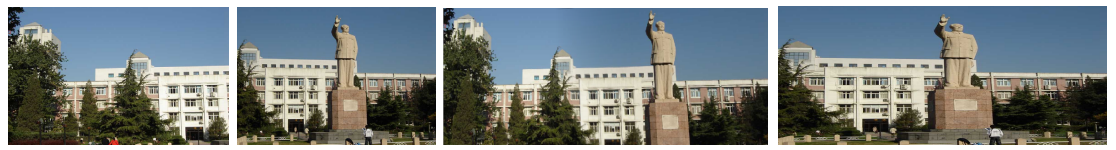

Fig. 4. Original images (left two images) and the image after stitching using our method (middle) and using software (right) 
The original images with obvious illumination difference are shown in the left two images of figure 4 . There are some commercial softwares to stitch panoramic images now in the market, so we also use one of them that can get good result to stitch the images to compare with ours, which is shown in the right image of figure 4 . From these results we can see a higher graphics resolution is obtained by using our method than by software.

The main purpose of panorama is to construct virtual environment. We apply the panorama to a computer game we are now exploiting about tank war and get higher game environment reality. In figure 5, the two tanks are modeled by java3D and the environment is constructed by panorama that is stitched by a series of images with overlapping region.
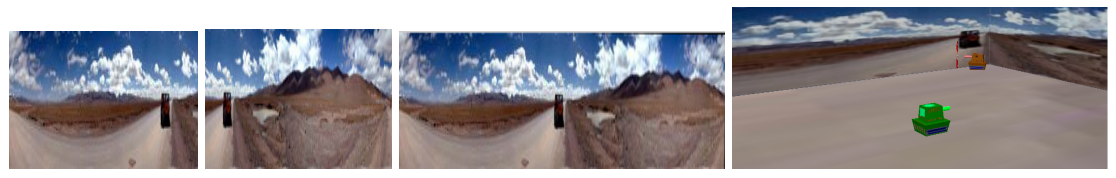

Fig. 5. The left two images are the original images and the middle is the panorama and the right is the game environment using panorama

\section{Conclusions}

Some experiment results have been presented using SIFT feature-based algorithm in this paper. From these results, we can see that using SIFT features to transform images allows us to stitch them in an accurate way. In addition, because we stitch the images by using the coordinates of the image after transformation instead of interpolation, unnecessary calculation is avoided and the transition region is detected easily. Besides, the approach that is to add adjacent images by weight function is simple and effective.

\section{Acknowledgments}

This work is supported by National 973 Key Research Program 2006CB303105, National 973 Key Research Program 2004CB318110 and University Key Research Fund 2004SZ002.

\section{References}

1. Shum, H.Y., Szelishi, R.: Panoramic Image Mosaics. Technical Report, Microsoft Research (1998)

2. Kang, G.: Virtual Environment Touring based on image, master thesis, Nanjing Institute of Technology (2004)

3. Hartley, R., Zisserman, A.: Multiple View Geometry in Computer Vision. AnHui University Press (2002)

4. Lowe, D.: Distinctive image features from scale-invariant keypoints. Int. Journal of Computer Vision 60(2), 91-110 (2004) 
5. Zhao, H.: Matching Technology based on SIFT Features, Shan Dong University, Institute of Information Science and Engineering (2006)

6. Efros, A., Freeman, W.: Image quilting for texture synthesis and transfer. In: Proc. SIGGRAPH, pp. 341-346 (August 2001)

7. Peleg, S.: Elimination of seams from photomosaics. Computer Graph Image Process. 16(1), 90-94 (1981)

8. Uyttendaele, M., Eden, A.: Eliminating ghosting and exposure artifacts in image mosaics. In: Proc. Conf. Computer Vision and Pattern Recognition, pp. II: 509-II: 516 (2001) 